\title{
Bacterial and archaeal diversity and abundance in different clay layers of subsurface at Jianghan Plain, China
}

\author{
DANDAN SONG, ZHOU JiANG AND LIANG SHI
}

Department of Biological Sciences and Technology, School of Environmental Studies, China University of Geosciences, Wuhan, Hubei 430074, People's Republic of China. liang.shi@cug.edu.cn

We analyzed the bacterial and archaeal community structure and abundance of six clay sediments at different depths in a borehole from Jianghan Plain $\left(112^{\circ} 34^{\prime} 0^{\prime \prime} \mathrm{E}\right.$ $\left.30^{\circ} 36^{\prime} 21^{\prime \prime} \mathrm{N}\right)$ via $16 \mathrm{~s}$ rRNA gene high throughput sequencing and q-PCR. The microbial community identified from all clay sediments was comprised of $67.5 \%$ bacterial phyla, such as Chloroflexi (17.5\%), Proteobacteria (11.7\%), Acidobacteria (7.1\%), Planctomycetes (4.0\%), Actinobacteria (3.7\%) and NC10 (2.6\%), and $29.5 \%$ of archaeal phyla, which included Bathyarchaeota (16.2\%), Euryarchaeota (6.1\%), Thaumarchaeota (3.9\%) and Nanoarchaeaeota (3.3\%). Both sequencing and q-PCR results consistently showed a higher ratio of archaea to bacteria in the bottom clay layers, as compared to that at the top clay layers. Furthermore, distinctly vertical stratification of microbial communities in these clay sediments was evident, which was mainly shaped by sulfate, iron and total organic carbon (TOC) concentrations in the different clay layers. In the upper clay layers, identification of Thaumarchaeota $(11 \%)$ and $\mathrm{NC10}$ $(6 \%)$ phyla indicated possible microbial ammonium oxidation and nitrate-dependent anaerobic methane oxidation in these layers. In the middle clay layers, a portion of identified microorganisms were obligate fermenters and iron reducers, such as Geobacter and Anaeromyxobacter. The latter suggests occurrence of bacterial dissimilatory iron reduction in these layers. Remarkably, a significant portion of Bathyarchaeota $(\sim 25 \%)$ that mainly consisted of subgroup 6 and subgroup 12 inhabited in the bottom clay layers, which suggest archaeal anaerobic degradation of TOC and possible sulfate reduction and methane formation in these layers. The results of this study not only advance our understandings of bacterial and archaeal community structure and abundances in different clay layers at subsurface, but also lay the foundation for future investigation of bacterial and archaeal roles in biogeochemical development of water-impermeable aquitard from water-permeable and -semipermeable clay layers. 